\title{
Short-term Femara (letrozole) treatment and suppression of Ki67 expression in postmenopausal endometrial carcinoma"
}

\author{
Lloyd H. Smith", Gary Leiserowitz, Guibo Xing, John Bishop \\ Department of Obstetrics, Gynecology and Pathology, UC Davis School of Medicine Sacramento, Sacramento, USA \\ Email: ${ }^{\# 1 h s m i t h @ u c d a v i s . e d u ~}$
}

Received 2 March 2013; revised 3 April 2013; accepted 10 April 2013

Copyright (C) 2013 Lloyd H. Smith et al. This is an open access article distributed under the Creative Commons Attribution License, which permits unrestricted use, distribution, and reproduction in any medium, provided the original work is properly cited.

\begin{abstract}
Objective: To determine if short-term treatment with Femara (letrozole) induces measurable reduction in tumor Ki67 expression in postmenopausal women with FIGO grade 1 and 2 endometrial carcinoma. Methods: In this non-randomized prospective study, 12 patients were given Femara (letrozole) $2.5 \mathrm{mg}$ daily for approximately 3 weeks prior to planned hysterectomy for FIGO grade 1 or 2 endometrial carcinoma. A group of 12 demographically similar patients were enrolled as no-treatment controls. Ki67 expression in tumor cells was quantitated by immunohistochemistry with mechanical scanning within the initial endometrial biopsy and compared to that in the hysterectomy specimen for each patient in the treatment and control groups. Results: The treatment and control groups were similar in age, tumor grade and FIGO stage. When "aromatase inhibitor responsiveness" was defined as proportionate decline in Ki67\% stained tumor cells of at least $70 \%$ between the pre-treatment endometrial biopsy and post-treatment hysterectomy specimens, 5 of 12 patients were found to be "responsive" in the treatment group with none of the controls fulfilling these criteria. Conclusion: Femara (letrozole) $2.5 \mathrm{mg}$ daily for approximately 3 weeks induced a significant reduction in tumor cell Ki67 expression among 5 of 12 (41\%) postmenopausal women with FIGO grade 1 or 2 endometrial carcinoma. We postulate that Ki67 may be a useful marker during aromatase inhibitor medical treatment of patients with endometrial cancer who are not candidates for surgical treatment.
\end{abstract}

\footnotetext{
*Conflict of Interest: Novartis Corporation provided Femara to patients participating in this clinical trial at no cost; Novartis Corporation did not provide financial support to the investigators for this research project. The authors report no conflict of interest.

\#Corresponding author.
}

Keywords: Endometrial Carcinoma; Aromatase Inhibitor; Letrozole; Ki67

\section{INTRODUCTION}

In 2012, cancers of the uterine corpus remain the fourth most common incident malignancy and the eighth most frequent cause of cancer mortality among women in the USA [1]. The aging of the population [2] and the association of endometrial cancer with certain medical comorbidities results in many such cases presenting with advanced age, declining functional status or serious concurrent medical illness. Although hysterectomy remains the initial treatment recommendation for most patients, women with endometrial cancer who are old and frail, or who have other serious medical conditions, experience increased risk of perioperative morbidity and mortality [3-5]. Consequently, effective medical treatments are needed as an alternative to surgery for the oldest and sickest women with endometrial cancer. The development of aromatase inhibitors (AIs) for the treatment of breast cancer potentially provides such a therapeutic alternative since AIs block the synthesis of estradiol, depriving the estrogen receptor of its ligand which promotes proliferation of both breast and endometrial malignant neoplasms. Although there are no AIs in the USA which are currently FDA approved for treatment of endometrial cancer, their use for this purpose is rational and supported by previous clinical research observations. Berstein et al. [6] reported endometrial tumor shrinkage by ultrasound during brief letrozole treatment prior to hysterectomy in 10 patients with endometrial carcinoma. Others have also found tumor shrinkage and disease stabilization during palliative treatment with AIs [7,8], but some studies suggest AIs often are often ineffective, especially for endometrial cancers of high tumor grade [9]. For AIs to be utilized successfully, treatment effects need 
to be measurable. Miller et al. [10] demonstrated significant reductions in the expression of the nuclear proliferation marker Ki67 within serial breast tumor biopsies after short-term treatment with AIs. We reasoned that Ki67 expression may also be a useful marker to help with the monitoring and management of patients with endometrial cancer who are being given AI medical treatment. Here we report the results of a pilot clinical trial asking if short-term AI treatment of FIGO grade 1 or 2 endometrial carcinoma in postmenopausal women is associated with significant decline in tumor Ki67 expression.

\section{MATERIALS AND METHODS}

A clinical trial to observe the effects of pre-hysterectomy Femara (letrozole) treatment in postmenopausal women with FIGO grade 1 or 2 endometrial carcinoma was approved by the U C Davis Human Subjects Committee. Eligibility criteria included women age 60 or older, or ages 40 - 59 with a 12 month history of amenorrhea and a serum follicle stimulating hormone level in the postmenopausal range. Women exposed to exogenous estrogens, progestins or tamoxifen within 30 days of enrollment were excluded. A demographically similar group of untreated control subjects were recruited from the group of patients who declined to participate in letrozole treatment. Paraffin embedded endometrial tissue from the initial endometrial biopsy or dilation and curettage specimen (hereafter referred to generically as "EMB") was obtained for each patient, as was paraffin embedded endometrial tissue from each patient's hysterectomy specimen. Subjects in the treatment arm took Femara 2.5 mg daily until the day prior to scheduled hysterectomy. The number of days of treatment was determined by counting the Femara tablets returned at the time of admission for hysterectomy. The final pathologic diagnosis was determined after review of the hysterectomy specimen by two pathologists from the Department of Pathology at UC Davis.

\subsection{Immunohistochemistry}

Immunohistochemistry was performed on 6 micron deparaffinized sections from EMB and hysterectomy tissues using antibody MIB-1 (Dako) directed against Ki67 nuclear antigen. Blocking of endogenous peroxidase, antigen retrieval, secondary antibody and DAB staining were done as previously described [11]. Appropriate positive and negative controls were utilized. The stained tumor nuclei were counted mechanically by autoscanner (Aperio Spectrum V platform, version 9.1). Intensity threshold, pixel area, hue settings and color saturation threshold were chosen to give the best results when viewing positive and negative control slides and to pref- erentially delineate strongly stained nuclei from unstained or weakly stained cells. Representative fields were selected for scanning that showed intact tumor without necrosis. Fragmented areas, regions of associated hyperplasia, stroma-rich areas and tissue section defects were avoided. Each specimen was done in triplicate using non-adjacent sections from each paraffin block separated by approximately 60 microns. Results were expressed as follows: $100 \times$ (number of strongly positive cells)/(number of strongly positive plus number of negative cells). Overall, standard deviations were found to be $5 \%$ or less, except for two EMB specimens with higher variance, exclusion of which did not change the results of the analysis.

\subsection{Statistical Analysis}

The proportion of the treated and control groups showing proportionate $\mathrm{Ki} 67 \%$ reductions of at least $70 \%$ were analyzed using the Fisher's Exact test. The demographic characteristics between the treated and control groups were analyzed using Student's t-test or Fisher's Exact test. The Student's t-test was used to compare the average Ki67\% staining between the treated and control groups.

\section{RESULTS}

Twelve patients enrolled in this study completed Femara treatment and were found to have FIGO grade 1 or 2 endometrial carcinoma within their hysterectomy specimen. Five patients who completed short-term Femara treatment were excluded from this analysis: two had hysterectomy tissues showing areas of FIGO grade 3 disease; two were found to have no stromal invasive component within their endometrial neoplasm; one patient had no identifiable residual neoplasm. For the 12 patients included in this analysis, the average days of Femara treatment was 23.5 (range 14 - 36). Demographic characteristics between the treatment and control groups were similar (Table 1) with no significant differences in average age $(\mathrm{p}=0.58)$, BMI $(\mathrm{p}=0.26)$, tumor grade $(\mathrm{p}=$ $0.67)$ or stage $(p=1.0)$. During treatment, patients receiving Femara were contacted by telephone on a weekly basis and a symptom record kept. Most patients reported mild hot flashes during the second or third week of treatment. Based on the extensive profile of Femara from prior clinical trials, no unexpected symptoms were encountered among the patients reported here during this short-term trial.

Tumor tissue Ki67 counts for the EMB and hysterictomy tissues are displayed in the Table $\mathbf{1}$ as averages of triplicate measurements on three non-contiguous sections from each paraffin block. Heterogeneity in the distribution of Ki67 tumor cell staining was especially apparent 
Table 1. Demographics and Ki67 Results.

\begin{tabular}{|c|c|c|c|c|c|c|c|c|c|c|c|c|}
\hline Subject \# & Age & BMI & $\begin{array}{c}\text { Tx } \\
\text { Days }\end{array}$ & Grade & Stage & Ki67EMB & $\begin{array}{l}\text { std. } \\
\text { dev. }\end{array}$ & Ki67Hyst & std.dev. & $\begin{array}{l}\text { Absolute } \\
\text { Change }\end{array}$ & $\begin{array}{c}\text { Proportionate } \\
\text { Change }\end{array}$ & $\begin{array}{l}\text { "Responder } \\
\text { Status" }\end{array}$ \\
\hline 1 & 64 & 33.6 & 30 & 1 & $1 \mathrm{~A}$ & 15 & 3.2 & 2 & 0.2 & -13 & -86.7 & 1 \\
\hline 2 & 60 & 32.2 & 20 & 1 & $1 \mathrm{~A}$ & 12 & 0.7 & 3 & 0.1 & -9 & -75 & 1 \\
\hline 3 & 67 & 30.2 & 21 & 1 & $1 \mathrm{~A}$ & 10 & 3.4 & 3 & 1.5 & -7 & -70 & 1 \\
\hline 4 & 69 & 27 & 30 & 1 & $1 \mathrm{~A}$ & 8 & 2.4 & 3 & 0.3 & -5 & -62.5 & 0 \\
\hline 5 & 61 & 36.8 & 22 & 1 & $1 \mathrm{~A}$ & 10 & 2.7 & 13 & 2.2 & 3 & 33.3 & 0 \\
\hline 6 & 71 & 22.9 & 36 & 2 & $1 \mathrm{~B}$ & 18 & 11.6 & 8 & 3.7 & -10 & -55.6 & 0 \\
\hline 7 & 55 & 47.9 & 30 & 1 & $1 \mathrm{~A}$ & 15 & 6.4 & 2 & 0.6 & -13 & -86.7 & 1 \\
\hline 8 & 58 & 29.9 & 14 & 1 & $1 \mathrm{~A}$ & 24 & 5.3 & 16 & 2.8 & -8 & -33.3 & 0 \\
\hline 9 & 82 & 32.2 & 22 & 2 & $3 \mathrm{C}$ & 3 & 1.9 & 27 & 2.5 & 24 & 800 & 0 \\
\hline 10 & 54 & 45.9 & 20 & 1 & $1 \mathrm{~B}$ & 2 & 0.3 & 7 & 1.0 & 5 & 250 & 0 \\
\hline 11 & 71 & 36.8 & 15 & 1 & $1 \mathrm{~A}$ & 25 & 3.3 & 7 & 2.0 & -18 & -72 & 1 \\
\hline 12 & 73 & 40.1 & 22 & 2 & $1 \mathrm{~A}$ & 8 & 1.7 & 14 & 1.6 & 6 & 75 & 0 \\
\hline $\begin{array}{l}\text { Mean of treated } \\
\text { group (above) }\end{array}$ & 65.4 & 34.625 & & & & 12.5 & 3.575 & 8.75 & 1.5 & -3.75 & 51.375 & \\
\hline 13 & 70 & 28.5 & 0 & 1 & $1 \mathrm{~A}$ & 28 & 1.1 & 27 & 1.9 & -1 & -3.6 & 0 \\
\hline 14 & 72 & 44.4 & 0 & 1 & $1 \mathrm{~A}$ & 22 & 5.2 & 20 & 2.6 & -2 & -9.1 & 0 \\
\hline 15 & 57 & 43.5 & 0 & 1 & $1 \mathrm{~A}$ & 22 & 7 & 14 & 2.8 & -8 & -36.4 & 0 \\
\hline 16 & 60 & 33 & 0 & 2 & $1 \mathrm{~A}$ & 24 & 2.1 & 28 & 3.1 & 4 & 16.7 & 0 \\
\hline 17 & 61 & 48.6 & 0 & 1 & $1 \mathrm{~A}$ & 10 & 1.3 & 12 & 1.8 & 2 & 20 & 0 \\
\hline 18 & 70 & 41.9 & 0 & 1 & $1 \mathrm{~A}$ & 4 & 0.8 & 25 & 1.7 & 21 & 525 & 0 \\
\hline 19 & 66 & 40.2 & 0 & 2 & $1 \mathrm{~A}$ & 31 & 5.4 & 49 & 1.4 & 18 & 58.1 & 0 \\
\hline 20 & 63 & 35.9 & 0 & 2 & $1 \mathrm{~A}$ & 22 & 2.9 & 24 & 1.6 & 2 & 9.1 & 0 \\
\hline 21 & 61 & 36 & 0 & 1 & $1 \mathrm{~A}$ & 37 & 4.4 & 39 & 5.1 & 2 & 5.4 & 0 \\
\hline 22 & 67 & 45.3 & 0 & 1 & $1 \mathrm{~A}$ & 43 & 2.4 & 13 & 1.9 & -30 & -69.8 & 0 \\
\hline 23 & 58 & 25.2 & 0 & 2 & $3 \mathrm{C}$ & 9 & 0.5 & 16 & 2.4 & 7 & 77.8 & 0 \\
\hline 24 & 61 & 33.6 & 0 & 2 & 1B & 10 & 2.7 & 14 & 0.8 & 4 & 40 & 0 \\
\hline $\begin{array}{l}\text { Mean of untreated } \\
\text { group }\end{array}$ & 63.833 & 38.008 & & & & 21.833 & 2.983 & 23.417 & 2.241 & 1.583 & 52.767 & \\
\hline $\begin{array}{l}\text { P-value for testing } \\
\text { difference between } \\
\text { treated and } \\
\text { untreated group }\end{array}$ & $\mathrm{p}=0.577$ & \multicolumn{2}{|c|}{$\begin{array}{c}\text { T-test } \\
p=0.265\end{array}$} & $\begin{array}{c}\text { Fisher's } \\
\text { exact test } \\
\mathrm{p}=0.6668\end{array}$ & $\begin{array}{l}\text { Fisher's } \\
\text { exact test } \\
p=1.0\end{array}$ & & & & & $\begin{array}{c}\text { T-test } \\
\mathrm{p}=0.07\end{array}$ & & $\begin{array}{c}\text { Fisher's exact } \\
\text { test } \\
\mathrm{p}=0.0373\end{array}$ \\
\hline
\end{tabular}

in the hysterectomy specimen endometrial tissues and representative areas were chosen for scanning to best represent the average distribution throughout the specimen. The Ki67 expression among the treated patients decreased by an average of $3.8 \%$ from pre-treatment EMB to post-treatment hysterectomy, while the Ki67 expression between EMB and hysterectomy in the un- treated group increased an average of $1.6 \%(\mathrm{p}=0.07)$. As an alternative to comparing the absolute changes $\%$ Ki67 counts, we also examined differences in the proportionate decrease of Ki67 between pre-treatment EMB and post-treatment hysterectomy specimens for each patient in the trial. As can be seen from the Table 1, a subset of the treated patients showed large proportional 
reductions in Ki67 expression after treatment. If "aromatase responsiveness" was defined as a minimum of $70 \%$ proportionate decline in Ki67 expression between EMB and hysterectomy, 5 of 12 treated patients were so classified, while none of the control patients were in this category $(p=0.03)$. The average days of Femara taken by the 5 "responder" patients (23.2) were similar to the average treatment days for the "non-responder" group (23.7).

\section{DISCUSSION}

High potency progestins have been extensively studied in the setting of young women with endometrial carcinoma seeking treatments which allow preservation of fertility, and most knowledge of endocrine therapy for this disease comes from these studies [12]. More recently, AIs have been reported to have benefit for some patients with endometrial carcinoma who are too sick to withstand anesthesia and/or hysterectomy [8]. While use of AIs could avoid dangerous progestin-related side effects [13] and can in some cases be associated with disease resolution or prevention of disease progression, they often fail to have a benefit, especially in endometrial cancers with high grade characteristics [9]. Consequently when AIs are chosen to treat women with endometrial carcinoma, monitoring for treatment effect is needed. Ideally, treatment effect should be judged by serial endometrial biopsy, though for some very debilitated patients even office EMB could be challenging. However, the presence or absence of ahistologic response to endocrine therapy may take many months to become demonstrable, and during that time disease progression could occur in some patients who are destined to fail such treatment. A shortterm evaluation which could predict either treatment success (eventual complete histologic resolution of tumor) or failure (histologic persistence) within the first months of treatment would be valuable in the clinical care of sick women with endometrial cancer. Based on the results from the clinical trial presented here, proportionate changes in Ki67 expression could prove to be useful in this setting.

Utilizing Ki67 as a marker for AI responsiveness has limitations. We elected to employ machine scanning for measurement of Ki67 staining to minimize the variations associated with subjective analysis. There are also welldescribed micro-anatomical variations in Ki67 expression within endometrial carcinomas [14]. Proliferation and Ki67 expression are also more apparent at the invasive front of the tumor [15]. Consequently, the measured Ki67\% staining in a superficial biopsy could be variable depending on the area sampled, especially when compared to the more anatomically intact,full-thickness endometrium in a hysterectomy specimen. This phenomenon may help explain some of the variability we observed in comparing tumor Ki67 expression within en- dometrial biopsies to that of hysterectomy tissues. When comparing serial endometrial biopsies to each other, such variability, while still a concern, may prove to be less problematic. There are of course other intrinsic limitations to an endometrial biopsy since it represents only a small portion of the endometrial tumor. Examples of this were two patients in the treatment group eliminated from the analysis because their hysterectomy specimen contained unsuspected areas of high grade carcinoma, another phenomenon that makes treatment effect monitoring mandatory for women treated medically for endometrial carcinoma.

A large and sustained suppression of Ki67 expression in endometrial carcinoma tissues of postmenopausal women treated medically with AIs may be a harbinger for a beneficial outcome- either stable disease or histologic regression. Either may be a valued outcome for women unable to safely proceed to hysterectomy. The data presented in this pilot clinical trial support the feasibility of larger clinical trials aimed at refining the role of AIs in treatment of endometrial carcinoma in postmenopausal women who cannot safely have surgical treatment.

\section{ACKNOWLEDGEMENTS}

The authors thank Antoni Duleba MD for helpful discussions in the design of this clinical trial.

\section{REFERENCES}

[1] Siegel, R., Naishadham, D. and Jemal, A. (2012) Cancer statistics: 2012. CA: A Cancer Journal for Clinicians, 62, 10-29. doi:10.3322/caac. 20138

[2] Yanick, R. (2005) Population aging and cancer: A crossnational concern. Cancer Journal, 11, 437-441. doi:10.1097/00130404-200511000-00002

[3] Wright, J.D., Lewin, S.N., Barrena Medel, N.I., et al. (2011) Morbidity and mortality of surgery for endometrial cancer in the oldest old. American Journal of $\mathrm{Ob}$ stetrics and Gynecology, 205, 66.e1-66.e8.

[4] Kondalsamy-Chennakesavan, S., Bouman, C., DeJong, S., et al. (2009) Clinical audit in gynecological cancer surgery: Development of a risk scoring system to predict adverse events. Gynecologic Oncology, 115, 329-333. doi:10.1016/j.ygyno.2009.08.004

[5] Erekson, E., Yip, S., Ciarleglio, M., et al. (2011) Postoperative complications after gynecologic surgery. Obstetrics \& Gynecology, 118, 785-793. doi:10.1097/AOG.0b013e31822dac5d

[6] Berstein, L., Maximov, S., Gershfeld, E., et al. (2002) Neoadjuvant therapy of endometrial cancer with the aromatase inhibitor letrozole: Endocrine and clinical effects. European Journal of Obstetrics \& Gynecology and Reproductive Biology, 105, 161-165. doi:10.1016/S0301-2115(02)00147-1 
[7] Baker, L., Brand, I. and Crawford, S. (2009) Sustained effect of the aromatase inhibitors anastrozole and letrozole on endometrial thickness in patients with endometrial hyperplasia and endometrial carcinoma. Current Medical Research and Opinion, 25, 1105-1109. doi:10.1185/03007990902860549

[8] Altman, A., Thompson, J., Nelson, G., et al. (2012) Use of aromatase inhibitors as first- and second-line medical therapy in patients with endometrial adenocarcinoma: A retrospective study. Journal of Obstetrics and Gynaecology Canada, 34, 664-672.

[9] Rose, P., Brunetto, V.L., VanLe, L., et al. (2000) A phase II trial of anastrozole in advanced recurrent or persistent endometrial carcinoma: A Gynecologic Oncology Group study. Gynecologic Oncology, 78, 212-216. doi:10.1006/gyno.2000.5865

[10] Miller, W.R., White, S., Dixon, J.M., et al. (2006) Proliferation, steroid receptors and clinical/pathological response in breast cancer treated with letrozole. British Journal of Cancer, 94, 1051-1056. doi:10.1038/sj.bjc.6603001

[11] Sabattini, E., Bisgaard, K., Ascani, S., et al. (1998) The EnVision++ system: A new immunohistochemical method for diagnostics and research. Critical comparison with the APAAP, ChemMate, CSA, LABC, and SABC techniques. Journal of Clinical Pathology, 51, 506-511. doi:10.1136/jep.51.7.506

[12] Baker, J., Obermair, A., Gebski, V., et al. (2012) Efficacy of oral or intrauterine device-delivered progestin in patients with complex endometrial hyperplasia with atypia or early endometrial adenocarcinoma: A meta-analysis and systemic review of the literature. Gynecologic Oncology, 125, 263-270. doi:10.1016/j.ygyno.2011.11.043

[13] Marshall, L (2003) Megestrol acetate therapy in geriatric patients: Case reviews and associated deep vein thrombosis. The Consultant Pharmacist, 18, 764-773.

[14] Stewart, C., Crook, M. and Doherty, D. (2010) Microanatomical variation in cellular proliferation in endometrial adenocarcinoma, and inverse correlation between Ki67 and cytokeratin 7 expression. Histopathology, 57, 46-54. doi:10.1111/j.1365-2559.2010.03588.x

[15] Horree, N., Van Diest, P.J., Sie-Go, D., et al. (2007) The invasive front in endometrial carcinoma: Higher proliferation and associated derailment of cell cycle regulators. Human Pathology, 38, 1232-1238. doi:10.1016/j.humpath.2007.01.008 